\title{
Klein-Beltrami Model. Part II
}

\author{
Roland Coghetto \\ Rue de la Brasserie 5 \\ 7100 La Louvière, Belgium
}

\begin{abstract}
Summary. Tim Makarios (with Isabelle/HOL ${ }^{1}$ ) and John Harrison (with HOL-Light ${ }^{2}$ have shown that "the Klein-Beltrami model of the hyperbolic plane satisfy all of Tarski's axioms except his Euclidean axiom" [2, 3, 15, 4].

With the Mizar system [1, 10] we use some ideas are taken from Tim Makarios' MSc thesis 12 for formalized some definitions (like the tangent) and lemmas necessary for the verification of the independence of the parallel postulate. This work can be also treated as a further development of Tarski's geometry in the formal setting [9].
\end{abstract}

MSC: 51A05 51M10 03B35

Keywords: Tarski's geometry axioms; foundations of geometry; Klein-Beltrami model

MML identifier: BKMODEL2, version: 8.1.07 5.47.1318

\section{Beltrami-Cayley-Klein Disk Model}

The BK-model yielding a non empty subset of the projective space over $\mathcal{E}_{\mathrm{T}}^{3}$ is defined by the term

(Def. 1) the interior of the conic for $1,1,-1,0,0$ and 0.

Now we state the propositions:

(1) The BK-model misses the absolute.

(2) Let us consider an element $P$ of the projective space over $\mathcal{E}_{\mathrm{T}}^{3}$, and a non zero element $u$ of $\mathcal{E}_{\mathrm{T}}^{3}$. Suppose $P=$ the direction of $u$ and $P \in$ the BK-model. Then $u(3) \neq 0$.

\footnotetext{
1 https://www.isa-afp.org/entries/Tarskis_Geometry.html

2 https://github.com/jrh13/hol-light/blob/master/100/independence.ml 
Let $P$ be an element of the BK-model. The functor BK-to-REAL2 $(P)$ yielding an element of the inside of circle $(0,0,1)$ is defined by

(Def. 2) there exists a non zero element $u$ of $\mathcal{E}_{\mathrm{T}}^{3}$ such that the direction of $u=P$ and $u(3)=1$ and $i t=[u(1), u(2)]$.

Let $Q$ be an element of the inside of circle $(0,0,1)$. The functor $\operatorname{REAL2-to-BK}(Q)$ yielding an element of the BK-model is defined by

(Def. 3) there exists an element $P$ of $\mathcal{E}_{\mathrm{T}}^{2}$ such that $P=Q$ and $i t=$ the direction of $\left[(P)_{\mathbf{1}},(P)_{\mathbf{2}}, 1\right]$.

Now we state the propositions:

(3) Let us consider an element $P$ of the BK-model.

Then REAL2-to-BK(BK-to-REAL2 $(P))=P$.

Proof: Consider $u$ being a non zero element of $\mathcal{E}_{\mathrm{T}}^{3}$ such that the direction of $u=P$ and $u(3)=1$ and BK-to-REAL2 $(P)=[u(1), u(2)]$. Consider $Q$ being an element of $\mathcal{E}_{\mathrm{T}}^{2}$ such that $Q=$ BK-to-REAL2 $(P)$ and REAL2-to-BK(BK-to-REAL2 $(P))=$ the direction of $\left[(Q)_{\mathbf{1}},(Q)_{\mathbf{2}}, 1\right] .\left[(Q)_{\mathbf{1}}\right.$, $\left.(Q)_{2}, 1\right]$ and $u$ are proportional.

(4) Let us consider elements $P, Q$ of the BK-model. Then $P=Q$ if and only if BK-to-REAL2 $(P)=$ BK-to-REAL2 $(Q)$.

(5) Let us consider an element $Q$ of the inside of circle $(0,0,1)$. Then BK-to-REAL2(REAL2-to-BK $(Q))=Q$.

(6) Let us consider elements $P, Q$ of the BK-model, and elements $P_{1}, P_{2}$, $P_{3}$ of the absolute. Suppose $P \neq Q$ and $P_{1} \neq P_{2}$ and $P, Q$ and $P_{1}$ are collinear and $P, Q$ and $P_{2}$ are collinear and $P, Q$ and $P_{3}$ are collinear. Then

(i) $P_{3}=P_{1}$, or

(ii) $P_{3}=P_{2}$.

Proof: $P_{3}=P_{1}$ or $P_{3}=P_{2}$.

(7) Let us consider an element $P$ of the BK-model, an element $Q$ of the projective space over $\mathcal{E}_{\mathrm{T}}^{3}$, and a non zero element $v$ of $\mathcal{E}_{\mathrm{T}}^{3}$. Suppose $P \neq Q$ and $Q=$ the direction of $v$ and $v(3)=1$. Then there exists an element $P_{1}$ of the absolute such that $P, Q$ and $P_{1}$ are collinear.

Proof: Consider $u$ being a non zero element of $\mathcal{E}_{\mathrm{T}}^{3}$ such that the direction of $u=P$ and $u(3)=1$ and BK-to-REAL2 $(P)=[u(1), u(2)]$. Reconsider $s=[u(1), u(2)], t=[v(1), v(2)]$ as a point of $\mathcal{E}_{\mathrm{T}}^{2}$. Set $a=0$. Set $b=0$. Set $r=1$. Reconsider $S=s, T=t, X=[a, b]$ as an element of $\mathcal{R}^{2}$. Reconsider $w_{1}=\frac{-2 \cdot|(t-s, s-[a, b])|+\sqrt{\Delta\left(\sum\left(^{2}(T-S)\right), 2 \cdot|(t-s, s-[a, b])|, \sum\left(^{2}(S-X)\right)-r^{2}\right)}}{2 \cdot \sum^{2}(T-S)}$ as a real number. $s \neq t$. Consider $e_{1}$ being a point of $\mathcal{E}_{\mathrm{T}}^{2}$ such that 
$\left\{e_{1}\right\}=\operatorname{HalfLine}(s, t) \cap \operatorname{circle}(a, b, r)$ and $e_{1}=\left(1-w_{1}\right) \cdot s+w_{1} \cdot t$. Reconsider $f=\left[\left(e_{1}\right)_{1},\left(e_{1}\right)_{2}, 1\right]$ as an element of $\mathcal{E}_{\mathrm{T}}^{3}$. Reconsider $e_{3}=f$ as a non zero element of $\mathcal{E}_{\mathrm{T}}^{3} \cdot 1 \cdot e_{3}+\left(-\left(1-w_{1}\right)\right) \cdot u+\left(-w_{1}\right) \cdot v=0_{\mathcal{E}_{\mathrm{T}}^{3}}$.

(8) Let us consider an element $P$ of the BK-model, and a line $L$ of Inc-ProjSp (the real projective plane). Then there exists an element $Q$ of the projective space over $\mathcal{E}_{\mathrm{T}}^{3}$ such that

(i) $P \neq Q$, and

(ii) $Q \in L$.

(9) Let us consider real numbers $a, b, c, d, e$, and elements $u, v, w$ of $\mathcal{E}_{\mathrm{T}}^{3}$. Suppose $u=[a, b, e]$ and $v=[c, d, 0]$ and $w=[a+c, b+d, e]$. Then $\langle|u, v, w|\rangle=0$.

(10) Let us consider real numbers $a, b$, and a non zero real number $c$. Then $[a, b, c]$ is a non zero element of $\mathcal{E}_{\mathrm{T}}^{3}$.

(11) Let us consider elements $u, v$ of $\mathcal{E}_{\mathrm{T}}^{3}$, and real numbers $a, b, c, d, e$. Suppose $u=[a, b, c]$ and $v=[d, e, 0]$ and $u$ and $v$ are proportional. Then $c=0$.

(12) Let us consider elements $P, Q, R$ of the projective space over $\mathcal{E}_{\mathrm{T}}^{3}$, and non zero elements $u, v, w$ of $\mathcal{E}_{\mathrm{T}}^{3}$. Suppose $P=$ the direction of $u$ and $Q=$ the direction of $v$ and $R=$ the direction of $w$ and $(u)_{\mathbf{3}} \neq 0$ and $(v)_{\mathbf{3}}=0$ and $w=\left[(u)_{\mathbf{1}}+(v)_{\mathbf{1}},(u)_{\mathbf{2}}+(v)_{\mathbf{2}},(u)_{\mathbf{3}}\right]$. Then

(i) $R \neq P$, and

(ii) $R \neq Q$.

(13) Let us consider a line $L$ of Inc-ProjSp(the real projective plane), and elements $P, Q$ of the projective space over $\mathcal{E}_{\mathrm{T}}^{3}$. If $P \neq Q$ and $P, Q \in L$, then $L=\operatorname{Line}(P, Q)$.

(14) Let us consider a line $L$ of Inc-ProjSp(the real projective plane), elements $P, Q$ of the projective space over $\mathcal{E}_{\mathrm{T}}^{3}$, and non zero elements $u, v$ of $\mathcal{E}_{\mathrm{T}}^{3}$. Suppose $P, Q \in L$ and $P=$ the direction of $u$ and $Q=$ the direction of $v$ and $(u)_{\mathbf{3}} \neq 0$ and $(v)_{\mathbf{3}}=0$. Then

(i) $P \neq Q$, and

(ii) the direction of $\left[(u)_{\mathbf{1}}+(v)_{\mathbf{1}},(u)_{\mathbf{2}}+(v)_{\mathbf{2}},(u)_{\mathbf{3}}\right] \in L$.

Proof: $P \neq Q$. Reconsider $w=\left[(u)_{\mathbf{1}}+(v)_{\mathbf{1}},(u)_{\mathbf{2}}+(v)_{\mathbf{2}},(u)_{\mathbf{3}}\right]$ as a non zero element of $\mathcal{E}_{\mathrm{T}}^{3} \cdot\langle|u, v, w|\rangle=0$.

(15) Let us consider elements $u, v, w$ of $\mathcal{E}_{\mathrm{T}}^{3}$. Suppose $(v)_{\mathbf{3}}=0$ and $w=$ $\left[(u)_{\mathbf{1}}+(v)_{\mathbf{1}},(u)_{\mathbf{2}}+(v)_{\mathbf{2}},(u)_{\mathbf{3}}\right]$. Then $\langle|u, v, w|\rangle=0$.

(16) Let us consider a line $L$ of Inc-ProjSp(the real projective plane), an element $P$ of the projective space over $\mathcal{E}_{\mathrm{T}}^{3}$, and a non zero element $u$ of $\mathcal{E}_{\mathrm{T}}^{3}$. 
Suppose $P=$ the direction of $u$ and $P \in L$ and $u(3) \neq 0$. Then there exists an element $Q$ of the projective space over $\mathcal{E}_{\mathrm{T}}^{3}$ and there exists a non zero element $v$ of $\mathcal{E}_{\mathrm{T}}^{3}$ such that $Q=$ the direction of $v$ and $Q \in L$ and $P \neq Q$ and $v(3) \neq 0$. The theorem is a consequence of (15).

(17) Let us consider an element $P$ of the BK-model, and a line $L$ of Inc-ProjSp (the real projective plane). Suppose $P \in L$. Then there exists an element $Q$ of the projective space over $\mathcal{E}_{\mathrm{T}}^{3}$ such that

(i) $P \neq Q$, and

(ii) $Q \in L$, and

(iii) for every non zero element $u$ of $\mathcal{E}_{\mathrm{T}}^{3}$ such that $Q=$ the direction of $u$ holds $u(3) \neq 0$.

The theorem is a consequence of (16).

(18) Let us consider non zero elements $u, v$ of $\mathcal{E}_{\mathrm{T}}^{3}$, and a non zero real number $k$. Suppose $u=k \cdot v$. Then the direction of $u=$ the direction of $v$.

(19) Let us consider an element $P$ of the BK-model, and an element $Q$ of the projective space over $\mathcal{E}_{\mathrm{T}}^{3}$. Suppose $P \neq Q$. Then there exists an element $P_{1}$ of the absolute such that $P, Q$ and $P_{1}$ are collinear.

Proof: Reconsider $L=\operatorname{Line}(P, Q)$ as a line of Inc-ProjSp(the real projective plane). Consider $R$ being an element of the projective space over $\mathcal{E}_{\mathrm{T}}^{3}$ such that $P \neq R$ and $R \in L$ and for every non zero element $u$ of $\mathcal{E}_{\mathrm{T}}^{3}$ such that $R=$ the direction of $u$ holds $u(3) \neq 0$. Consider $u$ being a non zero element of $\mathcal{E}_{\mathrm{T}}^{3}$ such that the direction of $u=P$ and $u(3)=1$ and BK-to-REAL2 $(P)=[u(1), u(2)]$. Consider $v^{\prime}$ being an element of $\mathcal{E}_{\mathrm{T}}^{3}$ such that $v^{\prime}$ is not zero and the direction of $v^{\prime}=R$. Reconsider $k=\frac{1}{\left(v^{\prime}\right)_{3}}$ as a non zero real number. $k \cdot v^{\prime}$ is not zero. Reconsider $v=k \cdot v^{\prime}$ as a non zero element of $\mathcal{E}_{\mathrm{T}}^{3}$. the direction of $v=R$ and $v(3)=1$. Reconsider $s=[u(1), u(2)], t=[v(1), v(2)]$ as a point of $\mathcal{E}_{\mathrm{T}}^{2}$. Set $a=0$. Set $b=0$. Set $r=1$. Reconsider $S=s, T=t, X=[a, b]$ as an element of $\mathcal{R}^{2}$. Reconsider $w_{1}=\frac{-2 \cdot|(t-s, s-[a, b])|+\sqrt{\Delta\left(\sum\left(^{2}(T-S)\right), 2 \cdot|(t-s, s-[a, b])|, \sum\left(^{2}(S-X)\right)-r^{2}\right)}}{2 \cdot \sum\left(^{2}(T-S)\right.}$ as a real number. $s \neq t$. Consider $e_{1}$ being a point of $\mathcal{E}_{\mathrm{T}}^{2}$ such that $\left\{e_{1}\right\}=\operatorname{HalfLine}(s, t) \cap \operatorname{circle}(a, b, r)$ and $e_{1}=\left(1-w_{1}\right) \cdot s+w_{1} \cdot t$. Reconsider $f=\left[\left(e_{1}\right)_{1},\left(e_{1}\right)_{\mathbf{2}}, 1\right]$ as an element of $\mathcal{E}_{\mathrm{T}}^{3}$. Reconsider $e_{3}=f$ as a non zero element of $\mathcal{E}_{\mathrm{T}}^{3} \cdot 1 \cdot e_{3}+\left(-\left(1-w_{1}\right)\right) \cdot u+\left(-w_{1}\right) \cdot v=0_{\mathcal{E}_{\mathrm{T}}^{3}}$.

(20) Let us consider elements $P, Q$ of the BK-model. Suppose $P \neq Q$. Then there exist elements $P_{1}, P_{2}$ of the absolute such that

(i) $P_{1} \neq P_{2}$, and

(ii) $P, Q$ and $P_{1}$ are collinear, and 
(iii) $P, Q$ and $P_{2}$ are collinear.

Proof: Consider $u$ being a non zero element of $\mathcal{E}_{\mathrm{T}}^{3}$ such that the direction of $u=P$ and $u(3)=1$ and BK-to-REAL2 $(P)=[u(1), u(2)]$. Consider $v$ being a non zero element of $\mathcal{E}_{\mathrm{T}}^{3}$ such that the direction of $v=$ $Q$ and $v(3)=1$ and BK-to-REAL2 $(Q)=[v(1), v(2)]$. Reconsider $s=$ $[u(1), u(2)], t=[v(1), v(2)]$ as a point of $\mathcal{E}_{\mathrm{T}}^{2}$. Set $a=0$. Set $b=0$. Set $r=1$. Reconsider $S=s, T=t, X=[a, b]$ as an element of $\mathcal{R}^{2}$. Reconsider $w_{1}=\frac{-2 \cdot|(t-s, s-[a, b])|+\sqrt{\Delta\left(\sum\left(^{2}(T-S)\right), 2 \cdot|(t-s, s-[a, b])|, \sum\left({ }^{2}(S-X)\right)-r^{2}\right)}}{\left.2 \cdot\left(\sum{ }^{2}(T-S)\right)\right)}$ as a real number. Consider $e_{1}$ being a point of $\mathcal{E}_{\mathrm{T}}^{2}$ such that $\left\{e_{1}\right\}=$ HalfLine $(s, t) \cap \operatorname{circle}(a, b, r)$ and $e_{1}=\left(1-w_{1}\right) \cdot s+w_{1} \cdot t$. Reconsider $w_{2}=\frac{-2 \cdot|(s-t, t-[a, b])|+\sqrt{\Delta\left(\sum\left(^{2}(S-T)\right), 2 \cdot|(s-t, t-[a, b])|, \sum\left(^{2}(T-X)\right)-r^{2}\right)}}{\left.2 \cdot\left(\sum{ }^{2}(S-T)\right)\right)}$ as a real number. Consider $e_{2}$ being a point of $\mathcal{E}_{\mathrm{T}}^{2}$ such that $\left\{e_{2}\right\}=\operatorname{HalfLine}(t, s) \cap$ circle $(a, b, r)$ and $e_{2}=\left(1-w_{2}\right) \cdot t+w_{2} \cdot s$. Reconsider $f=\left[\left(e_{1}\right)_{\mathbf{1}},\left(e_{1}\right)_{\mathbf{2}}\right.$, $1]$ as an element of $\mathcal{E}_{\mathrm{T}}^{3}$. Reconsider $e_{3}=f$ as a non zero element of $\mathcal{E}_{\mathrm{T}}^{3}$. Reconsider $P_{1}=$ the direction of $e_{3}$ as a point of the projective space over $\mathcal{E}_{\mathrm{T}}^{3} \cdot 1 \cdot e_{3}+\left(-\left(1-w_{1}\right)\right) \cdot u+\left(-w_{1}\right) \cdot v=0_{\mathcal{E}_{\mathrm{T}}^{3}}$. Reconsider $g=\left[\left(e_{2}\right)_{\mathbf{1}},\left(e_{2}\right)_{\mathbf{2}}\right.$, $1]$ as an element of $\mathcal{E}_{\mathrm{T}}^{3}$. Reconsider $e_{4}=g$ as a non zero element of $\mathcal{E}_{\mathrm{T}}^{3}$. Reconsider $P_{2}=$ the direction of $e_{4}$ as a point of the projective space over $\mathcal{E}_{\mathrm{T}}^{3} \cdot 1 \cdot e_{4}+\left(-\left(1-w_{2}\right)\right) \cdot v+\left(-w_{2}\right) \cdot u=0_{\mathcal{E}_{\mathrm{T}}^{3}} \cdot P_{1} \neq P_{2}$.

(21) Let us consider elements $P, Q, R$ of the real projective plane, non zero elements $u, v, w$ of $\mathcal{E}_{\mathrm{T}}^{3}$, and real numbers $a, b, c, d$. Suppose $P \in$ the BK-model and $Q \in$ the absolute and $P=$ the direction of $u$ and $Q=$ the direction of $v$ and $R=$ the direction of $w$ and $u=[a, b, 1]$ and $v=[c, d, 1]$ and $w=\left[\frac{a+c}{2}, \frac{b+d}{2}, 1\right]$. Then

(i) $R \in$ the BK-model, and

(ii) $R \neq P$, and

(iii) $P, R$ and $Q$ are collinear.

Proof: Reconsider $P_{6}=P$ as an element of the BK-model. Consider $u_{2}$ being a non zero element of $\mathcal{E}_{\mathrm{T}}^{3}$ such that the direction of $u_{2}=P_{6}$ and $u_{2}(3)=1$ and BK-to-REAL2 $\left(P_{6}\right)=\left[u_{2}(1), u_{2}(2)\right]$. Consider $p$ being a point of $\mathcal{E}_{\mathrm{T}}^{2}$ such that $[v(1), v(2)]=p$ and $|p-[0,0]|=1$. Reconsider $R_{1}=[w(1), w(2)]$ as an element of $\mathcal{E}_{\mathrm{T}}^{2} \cdot\left|R_{1}-[0,0]\right|^{2}<1$. Consider $P_{1}$ being an element of $\mathcal{E}_{\mathrm{T}}^{2}$ such that $P_{1}=R_{1}$ and REAL2-to-BK $\left(R_{1}\right)=$ the direction of $\left[\left(P_{1}\right)_{\mathbf{1}},\left(P_{1}\right)_{\mathbf{2}}, 1\right] . P \neq R$ by [13, (29)].

(22) Let us consider elements $P, Q$ of the real projective plane. Suppose $P \in$ the absolute and $Q \in$ the BK-model. Then there exists an element $R$ of the real projective plane such that 
(i) $R \in$ the BK-model, and

(ii) $Q \neq R$, and

(iii) $R, Q$ and $P$ are collinear.

The theorem is a consequence of (21).

(23) Let us consider a line $L$ of Inc-ProjSp(the real projective plane), points $p, q$ of Inc-ProjSp(the real projective plane), and elements $P, Q$ of the real projective plane. Suppose $p=P$ and $q=Q$ and $P \in$ the BK-model and $Q \in$ the absolute and $q$ lies on $L$ and $p$ lies on $L$. Then there exist points $p_{1}, p_{2}$ of Inc-ProjSp(the real projective plane) and there exist elements $P_{1}, P_{2}$ of the real projective plane such that $p_{1}=P_{1}$ and $p_{2}=P_{2}$ and $P_{1} \neq P_{2}$ and $P_{1}, P_{2} \in$ the absolute and $p_{1}$ lies on $L$ and $p_{2}$ lies on $L$. The theorem is a consequence of (1), (22), and (20).

(24) Let us consider an element $P$ of the BK-model, and an element $Q$ of the absolute. Then there exists an element $R$ of the absolute such that

(i) $Q \neq R$, and

(ii) $Q, P$ and $R$ are collinear.

The theorem is a consequence of (1) and (23).

(25) Let us consider an element $P$ of the BK-model, and a non zero element $u$ of $\mathcal{E}_{\mathrm{T}}^{3}$. Suppose $P=$ the direction of $u$ and $u(3)=1$. Then $(u(1))^{2}+$ $(u(2))^{2}<1$.

(26) Let us consider elements $P_{1}, P_{2}$ of the absolute, an element $Q$ of the BKmodel, and non zero elements $u, v, w$ of $\mathcal{E}_{\mathrm{T}}^{3}$. Suppose the direction of $u=P_{1}$ and the direction of $v=P_{2}$ and the direction of $w=Q$ and $u(3)=1$ and $v(3)=1$ and $w(3)=1$ and $v(1)=-u(1)$ and $v(2)=-u(2)$ and $P_{1}, Q$ and $P_{2}$ are collinear. Then there exists a real number $a$ such that

(i) $-1<a<1$, and

(ii) $w(1)=a \cdot u(1)$, and

(iii) $w(2)=a \cdot u(2)$.

The theorem is a consequence of (25). 


\section{TANGEnT}

Let $P$ be an element of the absolute. The functor PoleInfty $(P)$ yielding an element of the real projective plane is defined by

(Def. 4) there exists a non zero element $u$ of $\mathcal{E}_{\mathrm{T}}^{3}$ such that $P=$ the direction of $u$ and $u(3)=1$ and $(u(1))^{2}+(u(2))^{2}=1$ and $i t=$ the direction of $[-u(2)$, $u(1), 0]$.

Now we state the propositions:

(27) Let us consider an element $P$ of the absolute. Then $P \neq \operatorname{PoleInfty}(P)$.

(28) Let us consider elements $P_{1}, P_{2}$ of the absolute. Suppose PoleInfty $\left(P_{1}\right)=$ PoleInfty $\left(P_{2}\right)$. Then

(i) $P_{1}=P_{2}$, or

(ii) there exists a non zero element $u$ of $\mathcal{E}_{\mathrm{T}}^{3}$ such that $P_{1}=$ the direction of $u$ and $P_{2}=$ the direction of $\left[-(u)_{1},-(u)_{\mathbf{2}}, 1\right]$ and $(u)_{\mathbf{3}}=1$.

Proof: Consider $u_{1}$ being a non zero element of $\mathcal{E}_{\mathrm{T}}^{3}$ such that $P_{1}=$ the direction of $u_{1}$ and $u_{1}(3)=1$ and $u_{1}(1)^{2}+u_{1}(2)^{2}=1$ and PoleInfty $\left(P_{1}\right)=$ the direction of $\left[-u_{1}(2), u_{1}(1), 0\right]$. Consider $u_{2}$ being a non zero element of $\mathcal{E}_{\mathrm{T}}^{3}$ such that $P_{2}=$ the direction of $u_{2}$ and $u_{2}(3)=1$ and $\left(u_{2}(1)\right)^{2}+\left(u_{2}(2)\right)^{2}=1$ and PoleInfty $\left(P_{2}\right)=$ the direction of $\left[-u_{2}(2)\right.$, $\left.u_{2}(1), 0\right]$. Reconsider $w_{1}=\left[-u_{1}(2), u_{1}(1), 0\right]$ as a non zero element of $\mathcal{E}_{\mathrm{T}}^{3}$. Reconsider $w_{2}=\left[-u_{2}(2), u_{2}(1), 0\right]$ as a non zero element of $\mathcal{E}_{\mathrm{T}}^{3}$. Consider $a$ being a real number such that $a \neq 0$ and $w_{1}=a \cdot w_{2}$. If $a=1$, then $P_{1}=P_{2}$. If $a=-1$, then there exists a non zero element $u$ of $\mathcal{E}_{\mathrm{T}}^{3}$ such that $P_{1}=$ the direction of $u$ and $P_{2}=$ the direction of $\left[-(u)_{1},-(u)_{2}, 1\right]$ and $(u)_{\mathbf{3}}=1$.

Let $P$ be an element of the absolute. The functor tangent $(P)$ yielding a line of the real projective plane is defined by

(Def. 5) there exists an element $p$ of the real projective plane such that $p=P$ and $i t=\operatorname{Line}(p$, PoleInfty $(P))$.

Let us consider an element $P$ of the absolute. Now we state the propositions:

(29) $P \in \operatorname{tangent}(P)$.

(30) tangent $(P) \cap$ (the absolute $)=\{P\}$.

Proof: $\{P\} \subseteq$ tangent $(P) \cap($ the absolute). tangent $(P) \cap($ the absolute $) \subseteq$ $\{P\}$.

(31) Let us consider elements $P_{1}, P_{2}$ of the absolute. If tangent $\left(P_{1}\right)=$ tangent $\left(P_{2}\right)$, then $P_{1}=P_{2}$. The theorem is a consequence of (30).

(32) Let us consider elements $P, Q$ of the absolute. Then there exists an element $R$ of the real projective plane such that 

(i) $R \in \operatorname{tangent}(P)$, and
(ii) $R \in \operatorname{tangent}(Q)$.

(33) Let us consider elements $P_{1}, P_{2}$ of the absolute. Suppose $P_{1} \neq P_{2}$. Then there exists an element $P$ of the real projective plane such that tangent $\left(P_{1}\right) \cap$ tangent $\left(P_{2}\right)=\{P\}$. The theorem is a consequence of $(31)$.

(34) Let us consider a square matrix $M$ over $\mathbb{R}$ of dimension 3 , an element $P$ of the absolute, an element $Q$ of the real projective plane, non zero elements $u, v$ of $\mathcal{E}_{\mathrm{T}}^{3}$, and finite sequences $f_{3}, f_{7}$ of elements of $\mathbb{R}$. Suppose $M=\operatorname{symmetric} 3(1,1,-1,0,0,0)$ and $P=$ the direction of $u$ and $Q=$ the direction of $v$ and $u=f_{3}$ and $v=f_{7}$ and $Q \in \operatorname{tangent}(P)$. Then SumAll QuadraticForm $\left(f_{7}, M, f_{3}\right)=0$.

Proof: Consider $p$ being an element of the real projective plane such that $p=P$ and tangent $(P)=\operatorname{Line}(p$, PoleInfty $(P))$. Consider $w$ being a non zero element of $\mathcal{E}_{\mathrm{T}}^{3}$ such that $P=$ the direction of $w$ and $w(3)=1$ and $(w(1))^{\mathbf{2}}+(w(2))^{\mathbf{2}}=1$ and $\operatorname{PoleInfty}(P)=$ the direction of $[-w(2), w(1)$, $0]$. Consider $a_{1}$ being a real number such that $a_{1} \neq 0$ and $w=a_{1} \cdot u$. $w(1)=a_{1} \cdot\left((u)_{1}\right)$ and $w(2)=a_{1} \cdot\left((u)_{2}\right)$ and $w(3)=a_{1} \cdot\left((u)_{3}\right)$. len $f_{3}=$ width $M$ and len $f_{7}=\operatorname{len} M$ and len $f_{3}=\operatorname{len} M$ and len $f_{7}=$ width $M$ and len $f_{3}>0$ and len $f_{7}>0$.

(35) Let us consider elements $P, Q, R$ of the absolute, and points $P_{1}, P_{2}, P_{3}$, $P_{4}$ of the real projective plane. Suppose $P, Q, R$ are mutually different and $P_{1}=P$ and $P_{2}=Q$ and $P_{3}=R$ and $P_{4} \in \operatorname{tangent}(P)$ and $P_{4} \in$ tangent $(Q)$. Then

(i) $P_{1}, P_{2}$ and $P_{3}$ are not collinear, and

(ii) $P_{1}, P_{2}$ and $P_{4}$ are not collinear, and

(iii) $P_{1}, P_{3}$ and $P_{4}$ are not collinear, and

(iv) $P_{2}, P_{3}$ and $P_{4}$ are not collinear.

Proof: $P_{4} \notin$ the absolute. Consider $p$ being an element of the real projective plane such that $p=P$ and $\operatorname{tangent}(P)=\operatorname{Line}(p, \operatorname{PoleInfty}(P))$. Consider $q$ being an element of the real projective plane such that $q=Q$ and tangent $(Q)=\operatorname{Line}(q, \operatorname{PoleInfty}(Q)) . P_{1}, P_{2}$ and $P_{4}$ are not collinear. $P_{1}, P_{3}$ and $P_{4}$ are not collinear. $P_{2}, P_{3}$ and $P_{4}$ are not collinear.

(36) Let us consider elements $P, Q$ of the absolute, an element $R$ of the real projective plane, and non zero elements $u, v, w$ of $\mathcal{E}_{\mathrm{T}}^{3}$. Suppose $P=$ the direction of $u$ and $Q=$ the direction of $v$ and $R=$ the direction of $w$ and $R \in \operatorname{tangent}(P)$ and $R \in \operatorname{tangent}(Q)$ and $u(3)=1$ and $v(3)=1$ and $w(3)=0$. Then

(i) $P=Q$, or 
(ii) $u(1)=-v(1)$ and $u(2)=-v(2)$.

The theorem is a consequence of (34).

(37) Let us consider an element $P$ of the absolute, an element $R$ of the real projective plane, and a non zero element $u$ of $\mathcal{E}_{\mathrm{T}}^{3}$. Suppose $R \in \operatorname{tangent}(P)$ and $R=$ the direction of $u$ and $u(3)=0$. Then $R=\operatorname{PoleInfty}(P)$. The theorem is a consequence of (34).

(38) Let us consider a non zero real number $a$, and an invertible square matrix $N$ over $\mathbb{R}_{\mathrm{F}}$ of dimension 3 . Suppose $N=\operatorname{symmetric} 3(a, a,-a, 0,0,0)$. Then (the homography of $N)^{\circ}$ (the absolute) $=$ the absolute.

Proof: (The homography of $N)^{\circ}$ (the absolute) $\subseteq$ the absolute by [8, (8)]. The absolute $\subseteq$ (the homography of $N)^{\circ}$ (the absolute) by [11, (4), (3)], [7, (89)].

(39) Let us consider a non zero element $r_{1}$ of $\mathbb{R}_{\mathrm{F}}$, and invertible square matrices $M, O$ over $\mathbb{R}_{\mathrm{F}}$ of dimension 3 . Suppose $O=\operatorname{symmetric} 3(1,1,-1,0,0,0)$ and $M=r_{1} \cdot O$. Then (the homography of $\left.M\right)^{\circ}($ the absolute $)=$ the absolute. Proof: $r_{1} \neq 0$ by [14, (34)].

(40) Let us consider an element $P$ of the absolute. Then tangent $(P)$ misses the BK-model. The theorem is a consequence of (29), (23), and (30).

(41) Let us consider elements $P, P_{3}, P_{4}$ of the real projective plane, elements $P_{1}, P_{2}$ of the absolute, and an element $Q$ of the real projective plane. Suppose $P_{1} \neq P_{2}$ and $P_{3}=P_{1}$ and $P_{4}=P_{2}$ and $P \in$ the BK-model and $P, P_{3}$ and $P_{4}$ are collinear and $Q \in \operatorname{tangent}\left(P_{1}\right)$ and $Q \in \operatorname{tangent}\left(P_{2}\right)$. Then there exists an element $R$ of the real projective plane such that

(i) $R \in$ the absolute, and

(ii) $P, Q$ and $R$ are collinear.

The theorem is a consequence of (40), (7), (37), (28), and (26).

(42) Let us consider elements $P, R, S$ of the real projective plane, and an element $Q$ of the absolute. Suppose $P \in$ the BK-model and $R \in \operatorname{tangent}(Q)$ and $P, S$ and $R$ are collinear and $R \neq S$. Then $Q \neq S$. The theorem is a consequence of (29), (23), and (30).

\section{Subgroup of $K$-Isometry}

Let $h$ be an element of EnsHomography3. We say that $h$ is $K$-isometry if and only if

(Def. 6) there exists an invertible square matrix $N$ over $\mathbb{R}_{\mathrm{F}}$ of dimension 3 such that $h=$ the homography of $N$ and (the homography of $N)^{\circ}($ the absolute $)=$ the absolute. 
Now we state the proposition:

(43) Let us consider an element $h$ of EnsHomography3.

Suppose $h=$ the homography of $I_{\mathbb{R}_{\mathrm{F}}}^{3 \times 3}$. Then $h$ is $K$-isometry.

The set of $K$-isometries yielding a non empty subset of EnsHomography3 is defined by the term

(Def. 7) $\{h$, where $h$ is an element of EnsHomography3 $: h$ is $K$-isometry $\}$.

The subgroup of $K$-isometries yielding a strict subgroup of GroupHomography3 is defined by

(Def. 8) the carrier of $i t=$ the set of $K$-isometries.

Now we state the propositions:

(44) Let us consider an element $h$ of the set of $K$-isometries, and an invertible square matrix $N$ over $\mathbb{R}_{\mathrm{F}}$ of dimension 3 . Suppose $h=$ the homography of $N$. Then (the homography of $N)^{\circ}$ (the absolute) $=$ the absolute.

(i) the homography of $I_{\mathbb{R}_{\mathrm{F}}}^{3 \times 3}=\mathbf{1}_{\text {GroupHomography3 }}$, and

(ii) the homography of $I_{\mathbb{R}_{\mathrm{F}}}^{3 \times 3}=\mathbf{1}_{\alpha}$, where $\alpha$ is the subgroup of $K$-isometries.

(46) Let us consider invertible square matrices $N_{1}, N_{2}$ over $\mathbb{R}_{\mathrm{F}}$ of dimension 3 , and elements $h_{1}, h_{2}$ of the subgroup of $K$-isometries. Suppose $h_{1}=$ the homography of $N_{1}$ and $h_{2}=$ the homography of $N_{2}$. Then

(i) $h_{1} \cdot h_{2}$ is an element of the subgroup of $K$-isometries, and

(ii) $h_{1} \cdot h_{2}=$ the homography of $N_{1} \cdot N_{2}$.

(47) Let us consider an invertible square matrix $N$ over $\mathbb{R}_{\mathrm{F}}$ of dimension 3 , and an element $h$ of the subgroup of $K$-isometries.

Suppose $h=$ the homography of $N$. Then

(i) $h^{-1}=$ the homography of $N^{\smile}$, and

(ii) the homography of $N^{\smile}$ is an element of the subgroup of $K$-isometries. The theorem is a consequence of (45).

(48) Let us consider an element $s$ of the projective space over $\mathcal{E}_{\mathrm{T}}^{3}$, and elements $p, q, r$ of the absolute. Suppose $p, q, r$ are mutually different and $s \in$ tangent $(p) \cap$ tangent $(q)$. Then there exists an invertible square matrix $N$ over $\mathbb{R}_{\mathrm{F}}$ of dimension 3 such that

(i) (the homography of $N)^{\circ}$ (the absolute) $=$ the absolute, and

(ii) (the homography of $N)(\operatorname{Dir} 101)=p$, and

(iii) (the homography of $N)(\operatorname{Dirm101})=q$, and

(iv) (the homography of $N)($ Dir011) $=r$, and 
(v) (the homography of $N)(\operatorname{Dir} 010)=s$.

Proof: Reconsider $P_{1}=p, P_{2}=q, P_{3}=r, P_{4}=s$ as a point of the real projective plane. $P_{1}, P_{2}$ and $P_{3}$ are not collinear and $P_{1}, P_{2}$ and $P_{4}$ are not collinear and $P_{1}, P_{3}$ and $P_{4}$ are not collinear and $P_{2}, P_{3}$ and $P_{4}$ are not collinear. Consider $N$ being an invertible square matrix over $\mathbb{R}_{\mathrm{F}}$ of dimension 3 such that (the homography of $\left.N\right)(\operatorname{Dir} 101)=P_{1}$ and (the homography of $N)\left(\right.$ Dirm101) $=P_{2}$ and (the homography of $N)(\operatorname{Dir} 011)=P_{3}$ and $($ the homography of $N)(\operatorname{Dir} 010)=P_{4}$. Consider $n_{1}$, $n_{2}, n_{3}, n_{4}, n_{5}, n_{6}, n_{7}, n_{8}, n_{9}$ being elements of $\mathbb{R}_{F}$ such that $N=\left\langle\left\langle n_{1}\right.\right.$, $\left.\left.n_{2}, n_{3}\right\rangle,\left\langle n_{4}, n_{5}, n_{6}\right\rangle,\left\langle n_{7}, n_{8}, n_{9}\right\rangle\right\rangle$. Reconsider $b=-1$ as an element of $\mathbb{R}_{\mathrm{F}}$. Reconsider $a=1$ as an element of $\mathbb{R}_{\mathrm{F}}$. Reconsider $a=1, b=-1$ as a non zero element of $\mathbb{R}_{\mathrm{F}}$. Reconsider $N_{1}=\langle\langle a, 0,0\rangle,\langle 0, a, 0\rangle,\langle 0,0$, $b\rangle\rangle$ as an invertible square matrix over $\mathbb{R}_{\mathrm{F}}$ of dimension 3 . Reconsider $M=N^{\mathrm{T}} \cdot N_{1} \cdot N$ as an invertible square matrix over $\mathbb{R}_{\mathrm{F}}$ of dimension 3 . Consider $v_{1}, v_{2}, v_{3}, v_{4}, v_{5}, v_{6}, v_{7}, v_{8}, v_{9}$ being elements of $\mathbb{R}_{\mathrm{F}}$ such that $M=\left\langle\left\langle v_{1}, v_{2}, v_{3}\right\rangle,\left\langle v_{4}, v_{5}, v_{6}\right\rangle,\left\langle v_{7}, v_{8}, v_{9}\right\rangle\right\rangle$. Reconsider $r_{1}=v_{1}, r_{2}=v_{2}$, $r_{3}=v_{3}, r_{4}=v_{5}, r_{5}=v_{6}, r_{6}=v_{9}$ as a real number. Consider $Q$ being a point of the projective space over $\mathcal{E}_{\mathrm{T}}^{3}$ such that $\operatorname{Dir} 101=Q$ and for every element $u$ of $\mathcal{E}_{\mathrm{T}}^{3}$ such that $u$ is not zero and $Q=$ the direction of $u$ holds qfconic $\left(r_{1}, r_{4}, r_{6}, 2 \cdot r_{2}, 2 \cdot r_{3}, 2 \cdot r_{5}, u\right)=0$. Consider $Q$ being a point of the projective space over $\mathcal{E}_{\mathrm{T}}^{3}$ such that $\operatorname{Dirm} 101=Q$ and for every element $u$ of $\mathcal{E}_{\mathrm{T}}^{3}$ such that $u$ is not zero and $Q=$ the direction of $u$ holds qfconic $\left(r_{1}, r_{4}, r_{6}, 2 \cdot r_{2}, 2 \cdot r_{3}, 2 \cdot r_{5}, u\right)=0$. Consider $Q$ being a point of the projective space over $\mathcal{E}_{\mathrm{T}}^{3}$ such that $\operatorname{Dir} 011=Q$ and for every element $u$ of $\mathcal{E}_{\mathrm{T}}^{3}$ such that $u$ is not zero and $Q=$ the direction of $u$ holds qfconic $\left(r_{1}, r_{4}, r_{6}, 2 \cdot r_{2}, 2 \cdot r_{3}, 2 \cdot r_{5}, u\right)=0 . r_{3}=0$ and $r_{1}=-r_{6}$ and $r_{2}=0$ and $r_{5}=0$ and $r_{1}=r_{4} \cdot r_{1} \neq 0$. (The homography of $M)^{\circ}($ the absolute $)=$ the absolute.

(49) Let us consider elements $p_{1}, q_{1}, r_{1}, p_{2}, q_{2}, r_{2}$ of the absolute, and elements $s_{1}, s_{2}$ of the real projective plane. Suppose $p_{1}, q_{1}, r_{1}$ are mutually different and $p_{2}, q_{2}, r_{2}$ are mutually different and $s_{1} \in \operatorname{tangent}\left(p_{1}\right) \cap \operatorname{tangent}\left(q_{1}\right)$ and $s_{2} \in$ tangent $\left(p_{2}\right) \cap$ tangent $\left(q_{2}\right)$. Then there exists an invertible square matrix $N$ over $\mathbb{R}_{\mathrm{F}}$ of dimension 3 such that

(i) (the homography of $N)^{\circ}$ (the absolute) $=$ the absolute, and

(ii) (the homography of $N)\left(p_{1}\right)=p_{2}$, and

(iii) (the homography of $N)\left(q_{1}\right)=q_{2}$, and

(iv) (the homography of $N)\left(r_{1}\right)=r_{2}$, and

(v) (the homography of $N)\left(s_{1}\right)=s_{2}$. 
The theorem is a consequence of (48) and (47).

(50) Let us consider elements $p_{1}, q_{1}, r_{1}, p_{2}, q_{2}, r_{2}$ of the absolute. Suppose $p_{1}, q_{1}, r_{1}$ are mutually different and $p_{2}, q_{2}, r_{2}$ are mutually different. Then there exists an invertible square matrix $N$ over $\mathbb{R}_{\mathrm{F}}$ of dimension 3 such that

(i) (the homography of $N)^{\circ}$ (the absolute) $=$ the absolute, and

(ii) (the homography of $N)\left(p_{1}\right)=p_{2}$, and

(iii) (the homography of $N)\left(q_{1}\right)=q_{2}$, and

(iv) (the homography of $N)\left(r_{1}\right)=r_{2}$.

The theorem is a consequence of (33), (48), and (47).

(51) Let us consider a collinearity space $C$, and elements $p, q, r, s$ of $C$. If Line $(p, q)=$ Line $(r, s)$, then $r, s$ and $p$ are collinear.

(52) Let us consider a collinearity space $C$, and elements $p, q$ of $C$. Then Line $(p, q)=\operatorname{Line}(q, p)$.

Proof: Line $(p, q) \subseteq \operatorname{Line}(q, p)$. Line $(q, p) \subseteq \operatorname{Line}(p, q)$.

(53) Let us consider an invertible square matrix $N$ over $\mathbb{R}_{\mathrm{F}}$ of dimension 3 , and elements $p, q, r, s$ of the projective space over $\mathcal{E}_{\mathrm{T}}^{3}$.

Suppose Line $(($ the homography of $N)(p)$, (the homography of $N)(q))=$ Line $(($ the homography of $N)(r)$, (the homography of $N)(s))$. Then

(i) $p, q$ and $r$ are collinear, and

(ii) $p, q$ and $s$ are collinear, and

(iii) $r, s$ and $p$ are collinear, and

(iv) $r, s$ and $q$ are collinear.

The theorem is a consequence of (51) and (52).

Let us consider an invertible square matrix $N$ over $\mathbb{R}_{\mathrm{F}}$ of dimension 3 and elements $p, q, r, s, t, u, n_{1}, n_{2}, n_{3}, n_{4}$ of the real projective plane. Now we state the propositions:

(54) Suppose $p \neq q$ and $r \neq s$ and $n_{1} \neq n_{2}$ and $n_{3} \neq n_{4}$ and $p, q$ and $t$ are collinear and $r, s$ and $t$ are collinear and $n_{1}=($ the homography of $N)(p)$ and $n_{2}=($ the homography of $N)(q)$ and $n_{3}=($ the homography of $N)(r)$ and $n_{4}=($ the homography of $N)(s)$ and $n_{1}, n_{2}$ and $u$ are collinear and $n_{3}, n_{4}$ and $u$ are collinear. Then

(i) $u=($ the homography of $N)(t)$, or

(ii) Line $\left(n_{1}, n_{2}\right)=\operatorname{Line}\left(n_{3}, n_{4}\right)$. 
(55) Suppose $p \neq q$ and $r \neq s$ and $n_{1} \neq n_{2}$ and $n_{3} \neq n_{4}$ and $p, q$ and $t$ are collinear and $r, s$ and $t$ are collinear and $n_{1}=$ (the homography of $N)(p)$ and $n_{2}=$ (the homography of $\left.N\right)(q)$ and $n_{3}=$ (the homography of $N)(r)$ and $n_{4}=($ the homography of $N)(s)$ and $n_{1}, n_{2}$ and $u$ are collinear and $n_{3}, n_{4}$ and $u$ are collinear and $p, q$ and $r$ are not collinear. Then $u=($ the homography of $N)(t)$. The theorem is a consequence of (54) and (53).

(56) Let us consider elements $p, q$ of the absolute, and elements $a, b$ of the BKmodel. Then there exists an invertible square matrix $N$ over $\mathbb{R}_{\mathrm{F}}$ of dimension 3 such that

(i) (the homography of $N)^{\circ}$ (the absolute) $=$ the absolute, and

(ii) (the homography of $N)(a)=b$, and

(iii) (the homography of $N)(p)=q$.

Proof: Consider $p^{\prime}$ being an element of the absolute such that $p \neq p^{\prime}$ and $p, a$ and $p^{\prime}$ are collinear. Consider $q^{\prime}$ being an element of the absolute such that $q \neq q^{\prime}$ and $q, b$ and $q^{\prime}$ are collinear. Consider $t$ being an element of the real projective plane such that tangent $(p) \cap$ tangent $\left(p^{\prime}\right)=\{t\}$. Consider $u$ being an element of the real projective plane such that tangent $(q) \cap$ tangent $\left(q^{\prime}\right)=\{u\}$. Reconsider $a^{\prime}=a$ as an element of the real projective plane. Consider $R_{1}$ being an element of the real projective plane such that $R_{1} \in$ the absolute and $a^{\prime}, t$ and $R_{1}$ are collinear. Reconsider $b^{\prime}=b$ as an element of the real projective plane. Consider $R_{2}$ being an element of the real projective plane such that $R_{2} \in$ the absolute and $b^{\prime}, u$ and $R_{2}$ are collinear. $p, p^{\prime}, R_{1}$ are mutually different. Consider $N$ being an invertible square matrix over $\mathbb{R}_{\mathrm{F}}$ of dimension 3 such that (the homography of $N)^{\circ}$ (the absolute) $=$ the absolute and (the homography of $\left.N\right)(p)=q$ and (the homography of $N)\left(p^{\prime}\right)=q^{\prime}$ and (the homography of $\left.N\right)\left(R_{1}\right)=R_{2}$ and (the homography of $N)(t)=u$. Reconsider $p_{5}=p, p_{6}=p^{\prime}, p_{7}=R_{1}$, $p_{8}=t, p_{9}=a, n_{1}=q, n_{2}=q^{\prime}, n_{3}=R_{2}, n_{4}=u, n_{5}=b$ as an element of the real projective plane. $n_{5}=($ the homography of $N)\left(p_{9}\right)$.

(57) Let us consider elements $p, q, r, s$ of the absolute. Suppose $p, q, r$ are mutually different and $q, p, s$ are mutually different. Then there exists an invertible square matrix $N$ over $\mathbb{R}_{\mathrm{F}}$ of dimension 3 such that

(i) (the homography of $N)^{\circ}$ (the absolute) $=$ the absolute, and

(ii) (the homography of $N)(p)=q$, and

(iii) (the homography of $N)(q)=p$, and

(iv) (the homography of $N)(r)=s$, and 
(v) for every element $t$ of the real projective plane such that $t \in \operatorname{tangent}(p) \cap$ tangent $(q)$ holds (the homography of $N)(t)=t$.

The theorem is a consequence of (33), (48), and (47).

Let us consider elements $P, Q$ of the BK-model. Now we state the propositions:

(58) Suppose $P \neq Q$. Then there exist elements $P_{1}, P_{2}, P_{3}, P_{4}$ of the absolute and there exists an element $P_{5}$ of the projective space over $\mathcal{E}_{\mathrm{T}}^{3}$ such that $P_{1} \neq P_{2}$ and $P, Q$ and $P_{1}$ are collinear and $P, Q$ and $P_{2}$ are collinear and $P, P_{5}$ and $P_{3}$ are collinear and $Q, P_{5}$ and $P_{4}$ are collinear and $P_{1}$, $P_{2}, P_{3}$ are mutually different and $P_{1}, P_{2}, P_{4}$ are mutually different and $P_{5} \in \operatorname{tangent}\left(P_{1}\right) \cap \operatorname{tangent}\left(P_{2}\right)$. The theorem is a consequence of (20), (32), (41), (30), (42), (29), (40), and (7).

(59) Suppose $P \neq Q$. Then there exists an invertible square matrix $N$ over $\mathbb{R}_{\mathrm{F}}$ of dimension 3 such that

(i) (the homography of $N)^{\circ}$ (the absolute) $=$ the absolute, and

(ii) (the homography of $N)(P)=Q$, and

(iii) (the homography of $N)(Q)=P$, and

(iv) there exist elements $P_{1}, P_{2}$ of the absolute such that $P_{1} \neq P_{2}$ and $P$, $Q$ and $P_{1}$ are collinear and $P, Q$ and $P_{2}$ are collinear and (the homography of $N)\left(P_{1}\right)=P_{2}$ and (the homography of $\left.N\right)\left(P_{2}\right)=P_{1}$.

Proof: Consider $P_{1}, P_{2}, P_{3}, P_{4}$ being elements of the absolute, $P_{5}$ being an element of the projective space over $\mathcal{E}_{\mathrm{T}}^{3}$ such that $P_{1} \neq P_{2}$ and $P, Q$ and $P_{1}$ are collinear and $P, Q$ and $P_{2}$ are collinear and $P, P_{5}$ and $P_{3}$ are collinear and $Q, P_{5}$ and $P_{4}$ are collinear and $P_{1}, P_{2}, P_{3}$ are mutually different and $P_{1}, P_{2}, P_{4}$ are mutually different and $P_{5} \in \operatorname{tangent}\left(P_{1}\right) \cap \operatorname{tangent}\left(P_{2}\right)$. Consider $N_{1}$ being an invertible square matrix over $\mathbb{R}_{\mathrm{F}}$ of dimension 3 such that (the homography of $\left.N_{1}\right)^{\circ}$ (the absolute) $=$ the absolute and (the homography of $\left.N_{1}\right)\left(\right.$ Dir101) $=P_{1}$ and (the homography of $\left.N_{1}\right)($ Dirm $101)=P_{2}$ and (the homography of $\left.N_{1}\right)\left(\right.$ Dir011) $=P_{3}$ and (the homography of $\left.N_{1}\right)\left(\right.$ Dir010) $=P_{5}$. Consider $N_{2}$ being an invertible square matrix over $\mathbb{R}_{\mathrm{F}}$ of dimension 3 such that (the homography of $\left.N_{2}\right)^{\circ}$ (the absolute) $=$ the absolute and (the homography of $\left.N_{2}\right)\left(\right.$ Dir101) $=P_{2}$ and (the homography of $\left.N_{2}\right)\left(\right.$ Dirm101) $=P_{1}$ and (the homography of $\left.N_{2}\right)($ Dir011 $)=P_{4}$ and (the homography of $\left.N_{2}\right)\left(\right.$ Dir010) $=P_{5}$. Reconsider $N=N_{2} \cdot\left(N_{1} \smile\right)$ as an invertible square matrix over $\mathbb{R}_{\mathrm{F}}$ of dimension 3 . Reconsider $h_{1}=$ the homography of $N_{1}$ as an element of EnsHomography3. Reconsider $h_{5}=h_{1}$ as an element of the subgroup of $K$-isometries. Reconsider $h_{2}=$ the homography of $N_{2}$ as an element of EnsHomography3. Reconsider 
$h_{6}=h_{2}$ as an element of the subgroup of $K$-isometries. Reconsider $h_{3}=$ the homography of $N_{1} \smile$ as an element of EnsHomography3. $h_{5}{ }^{-1}=h_{3}$. Reconsider $h_{7}=h_{3}$ as an element of the subgroup of $K$-isometries. Reconsider $h_{4}=h_{6} \cdot h_{7}$ as an element of the subgroup of $K$-isometries. Consider $h$ being an element of EnsHomography3 such that $h_{4}=h$ and $h$ is $K$-isometry. (the homography of $N)(P)=Q$ and (the homography of $N)(Q)=P$ by [ㅁ, (102), (57)], [6. (15)].

\section{Main Lemmas}

Now we state the propositions:

(60) Let us consider elements $P, Q$ of the BK-model. Then there exists an element $h$ of the subgroup of $K$-isometries and there exists an invertible square matrix $N$ over $\mathbb{R}_{\mathrm{F}}$ of dimension 3 such that $h=$ the homography of $N$ and (the homography of $N)(P)=Q$ and (the homography of $N)(Q)=P$. The theorem is a consequence of (43) and (59).

(61) Let us consider elements $P, Q, R, S, T, U$ of the BK-model. Suppose there exist elements $h_{1}, h_{2}$ of the subgroup of $K$-isometries and there exist invertible square matrices $N_{1}, N_{2}$ over $\mathbb{R}_{\mathrm{F}}$ of dimension 3 such that $h_{1}=$ the homography of $N_{1}$ and $h_{2}=$ the homography of $N_{2}$ and (the homography of $\left.N_{1}\right)(P)=R$ and (the homography of $\left.N_{1}\right)(Q)=S$ and (the homography of $\left.N_{2}\right)(R)=T$ and (the homography of $\left.N_{2}\right)(S)=U$. Then there exists an element $h_{3}$ of the subgroup of $K$-isometries and there exists an invertible square matrix $N_{3}$ over $\mathbb{R}_{\mathrm{F}}$ of dimension 3 such that $h_{3}=$ the homography of $N_{3}$ and (the homography of $\left.N_{3}\right)(P)=T$ and (the homography of $\left.N_{3}\right)(Q)=U$. The theorem is a consequence of (46).

(62) Let us consider elements $P, Q, R$ of the BK-model, an element $h$ of the subgroup of $K$-isometries, and an invertible square matrix $N$ over $\mathbb{R}_{\mathrm{F}}$ of dimension 3. Suppose $h=$ the homography of $N$ and (the homography of $N)(P)=R$ and (the homography of $N)(Q)=R$. Then $P=Q$.

\section{REFERENCES}

[1] Grzegorz Bancerek, Czesław Byliński, Adam Grabowski, Artur Korniłowicz, Roman Matuszewski, Adam Naumowicz, Karol Pąk, and Josef Urban. Mizar: State-of-the-art and beyond. In Manfred Kerber, Jacques Carette, Cezary Kaliszyk, Florian Rabe, and Volker Sorge, editors, Intelligent Computer Mathematics, volume 9150 of Lecture Notes in Computer Science, pages 261-279. Springer International Publishing, 2015. ISBN 978-3319-20614-1. doi 10.1007/978-3-319-20615-8_17.

[2] Eugenio Beltrami. Saggio di interpetrazione della geometria non-euclidea. Giornale di Matematiche, 6:284-322, 1868. 
[3] Eugenio Beltrami. Essai d'interprétation de la géométrie non-euclidéenne. In Annales scientifiques de l'École Normale Supérieure. Trad. par J. Hoüel, volume 6, pages 251288. Elsevier, 1869.

[4] Karol Borsuk and Wanda Szmielew. Podstawy geometrii. Państwowe Wydawnictwo Naukowe, Warszawa, 1955 (in Polish).

[5] Roland Coghetto. Homography in $\mathbb{R P}^{2}$. Formalized Mathematics, 24(4):239-251, 2016. doi: $10.1515 /$ forma-2016-0020.

[6] Roland Coghetto. Group of homography in real projective plane. Formalized Mathematics, 25(1):55-62, 2017. doi 10.1515/forma-2017-0005.

[7] Roland Coghetto. Klein-Beltrami model. Part I. Formalized Mathematics, 26(1):21-32, 2018. doi $10.2478 /$ forma-2018-0003.

[8] Roland Coghetto. Pascal's theorem in real projective plane. Formalized Mathematics, 25 (2):107-119, 2017. doi 10.1515/forma-2017-0011.

[9] Adam Grabowski. Tarski's geometry modelled in Mizar computerized proof assistant. In Proceedings of the 2016 Federated Conference on Computer Science and Information Systems, FedCSIS 2016, Gdańsk, Poland, September 11-14, 2016, pages 373-381, 2016. doi: $10.15439 / 2016$ F 290

[10] Adam Grabowski, Artur Korniłowicz, and Adam Naumowicz. Four decades of Mizar. Journal of Automated Reasoning, 55(3):191-198, 2015. doi:10.1007/s10817-015-9345-1

[11] Kanchun, Hiroshi Yamazaki, and Yatsuka Nakamura. Cross products and tripple vector products in 3-dimensional Euclidean space. Formalized Mathematıcs, 11(4):381-383, 2003.

[12] Timothy James McKenzie Makarios. A mechanical verification of the independence of Tarski's Euclidean Axiom. Victoria University of Wellington, New Zealand, 2012. Master's thesis.

[13] Yatsuka Nakamura. Graph theoretical properties of arcs in the plane and Fashoda Meet Theorem. Formalized Mathematıcs, $7(\mathbf{2}): 193-201,1998$.

[14] Karol Pąk and Andrzei Trybulec. Laplace expansion. Formalized Mathematics, 15(3): 143-150, 2007. doi 10.2478/v10037-007-0016-5

[15] Andrzej Trybulec. A Borsuk theorem on homotopy types. Formalized Mathematics, 2(4): 535-545, 1991. 